\title{
THE OPEN-CIRCUIT DECAY BEHAVIOUR OF CHLORINE-EVOLVING ELECTRODES
}

\author{
L. J. J. Janssen, G. J. Visser and E. Barendrecht \\ University of Technology, P.O. Box 513, 5600 MB Eindhoven, The Netherlands
}

\author{
(Received 30 April 1979)
}

\begin{abstract}
The open-circuit decay behaviour of a previously chlorine-evolving electrode, has been studied. It has been stated that the rate of the chlorine evolution on open circuit is determined by the parameters of the rate equation of the chlorine evolution and the diffusion of atomic chlorine out of the electrode. Both possible mechanisms viz. the Volmer-Tafel and the Volmer-Heyrovsky mechanism have been considered.

Numerically calculated Iela tions have been given between the overpotential on open circuit, the rate of the chlorine evolution on open circuit and time. Moreover, theoretical relations are calculated between the overpotential during the anodic polarisation and the overpotential on open circuit at the moment of, and $1 \mathrm{~s}$ after, switching off the polarising current, and the total quantity of chlorine evolved on open circuit, divided by the rate of the exchange reaction of the chlorine evolution.

Earlier experimental results on the behaviour on open circuit, of the chlorine chloride electrode in chloride melts and in aqueous acidic sodium chloride solutions have been compared with the calculated theoretical relations. It has been found that some earlier conclusions on the bchaviour of the chlorinc-chloride electrode need correction.
\end{abstract}

\section{NOMENCLATURE}

$c\left(x, t_{n}\right)$

concentration of atomic chlorine present in an electrode at a distance $x$ at time $t_{n}$ after switching off the polarisation current

$c_{0} \quad c(x, 0)$ at $x \geqslant 0$ or $c\left(\infty, t_{n}\right)$ at $t_{n} \geqslant 0$

$D$ diffusion coefficient of atomic chlorine

$E \quad$ electrode potential os sce

F Faraday constant

$h \quad$ the slope of the linear part of the $\eta_{n} / \log t_{n}$ curve

j real $c d$

$j_{0} \quad$ real exchange $c d$

$\dot{p} \quad$ constant factor : $p=\theta i c$

$\dot{q} \quad \int_{0}^{t_{m}} \frac{j_{n}}{j_{0}} \mathrm{~d} t_{m}$

$R \quad$ gas constant

$T \quad$ temperature

$t_{n} \quad$ time after switching off the polarisation current

$x \quad$ distance in the elestrode measured from the boundary electrode surface-electrolyte

Greek symbols

$\alpha$
$\eta$
$\eta_{n}$
$\eta_{n}(0)$
$\eta_{n}(1)$
$\theta$
$\theta_{0}$

\section{transfer coefficient}

overpotential during anodic polarisation overpotential on open circuit ; $\eta_{n}=E_{n}-E_{r}$ $\eta_{n}$ at $t_{n}=0$

$\eta_{n}$ at $t_{n}=1 \mathrm{~s}$

degree of coverage with atomic chlorine

$\theta$ at $t_{n}=0$; this being equal to the degree of coverage at the potential of the electrode on current flow

\section{Subscripts}

$\begin{array}{ll}i & \text { current flow conditions } \\ n & \text { open circuit conditions } \\ \boldsymbol{r} & \text { thermodynamic conditions } \\ \mathbf{H} & \text { Heyrovsky reaction } \\ \mathbf{T} & \text { Tafel reaction } \\ \mathbf{V} & \text { Volmer reaction }\end{array}$

\section{INTRODUCTION}

Investigation of the time-dependent decay of potential after polarisation of an electrode provides information complementary to that obtained by the investigation of potential-current density relations and its modifications. The potential decay behaviour can be used successfully to elucidate the mechanism of the electrode reactions [ $1-6]$.

Theoretically potential decay curves have been obtained by numerical calculations with computers. At practically the same time these calculations have been performed for the $\mathrm{H}_{2} / \mathrm{H}^{+}[3]$ and for the $\mathrm{Cl}_{2} / \mathrm{Cl}^{-}$ electrode process [4].

Tilak and Conway[3] have examined the potential decay behaviour assuming that the chemisorbed intermediate is only present at the surface of the electrode, whereas Janssen et al[4] have assumed that the chemisorbed intermediate is also present in the bulk of the electrode material by diffusion and diffuses out of this material during the potential decay. The diffusion of the intermediate out of the electrode material strongly affects potential decay behaviour[2, $4,7]$.

The calculated theoretical curves given in the literature are only useful at overpotentials greater than about $60 \mathrm{mV}$, owing to the neglect of the back reaction $[3,4]$. Also, the difference between the overpotential $\eta_{n}(0)$ on open circuit at the moment of switching off the polarising current $\left(t_{n}=0\right)$ and the overpotential $\eta_{n}$ on open circuit was calculated[4].

In the present paper, theoretical $\eta_{n} / t_{n}$-relations are derived for the $\mathrm{Cl}_{2} / \mathrm{Cl}^{-}$-process at $\eta_{n}>5 \mathrm{mV}$, the other conditions being the same as those in the literature [4]. Moreover, relations between overpotentials during the electrolysis and overpotential on open circuit are given.

\section{THEORY}

For an electrode process, involving chemisorbed intermediates, switching off the polarising current leads to a change of electrode potential $E$ with time $t_{n}$. The decay of electrode potential depends on both the discharge of the ionic double-layer and the decrease in 
the degree of coverage of the electrode surface by the intermediate of the electrode reactions.

Tilak and Conway[4] found that the effect of the double-layer capacitance upon the potential decay behaviour is insignificant. Their conclusion is surely correct for the case discussed here. The time constant for the discharge of the ionic double layer for an electrode process, not involving chemisorbed intermediates, is about $5 \times 10^{-3} \mathrm{~s}$ at an exchange-current density of about $0.1 \mathrm{~mA} / \mathrm{cm}^{2}[8]$.

In this paper the $\mathrm{Cl}_{2} / \mathrm{Cl}^{-}$-electrode process is considered. The results obtained can be applied to analogous processes for instance the $\mathrm{H}_{2} / \mathrm{H}^{+}$electrode process. The latter is generally discussed in the literature. The chlorine formation by the electrochemical oxidation of chloride ions can occur according to two different reaction mechanisms, viz the Volmer-Tafel mechanism and the Volmer-Heyrovsky mechanism.

It is assumed that, at the moment of switching off the polarisation current, atomic chlorine - the intermediate of the $\mathrm{Cl}_{2} / \mathrm{Cl}^{-}$electrode process - is also present in the bulk of the electrode material and reacts to molecular chlorine on the electrode surface according to both the Tafel and/or the Heyrovsky reaction.

\subsection{Volmer-Tafel mechanism}

The Volmer-Tafel mechanism consists of two reactions, viz the Volmer reaction $\left(\mathrm{Cl}^{-} \rightleftarrows \mathrm{Cl}_{\mathrm{ad}}+e^{-}\right)$ and the Tafel reaction $\left(2 \mathrm{Cl}_{\mathrm{ad}} \rightleftarrows \mathrm{Cl}_{2}\right.$ ). The potential on open circuit is determined by the equilibrium between the cathodic and the anodic Volmer reaction. The rate of the formation of molecular chlorine depends on the net rate of the Tafel reaction. The rate of the removal of atomic chlorine can be deduced from the rate equations of the Tafel reaction[9]. This rate can be expressed as a current density.

$$
j_{n, \mathrm{~T}}=j_{0, \mathrm{~T}}\left[\frac{\theta_{m}^{2}}{\theta_{r}^{2}}-\frac{\left(1-\theta_{\mu}\right)^{2}}{\left(1-\theta_{r}\right)^{2}}\right]
$$

It is assumed that the diffusion of chlorine atoms out of the bulk of the electrode material can be described as a one-dimensional diffusion process and satisfies the second equation of Fick.

$$
\frac{\delta c\left(x, t_{n}\right)}{\delta t_{n}}=D \frac{\delta^{2} c\left(x, t_{n}\right)}{\delta x^{2}}
$$

This equation is solved with the following boundary conditions

$$
\begin{gathered}
c(x, 0)=c_{0} \text { for } x \geq 0 \\
c\left(\infty, t_{n}\right)=c_{0} \text { for } t_{n} \geq 0 \\
D \frac{\delta c\left(x, t_{n}\right)}{\delta x}=\frac{j_{0} \mathrm{~T}}{F}\left(\frac{c^{2}\left(0, t_{n}\right)}{c_{r}^{2}}-\frac{\left(1-p c\left(0, t_{n}\right)\right)^{2}}{\left(1-p c_{r}\right)^{2}}\right)
\end{gathered}
$$

where $\theta=p c$ and $p$ is a constant factor. The latter boundary condition denotes that the rate of the diffusion of atomic chlorine out of the bulk of the electrode is equal to the rate of the removal of atomic chlorine on the electrode surface, see (1). The boundary conditions are the same as those in the literature with the exception of the last term on the right hand side. The additional term represents the infuence of the dissociation reaction of molecular chlorine upon the removal of atomic chlorine.

The relation between $c\left(0, t_{n}\right) / c_{r}$ and $t_{n}$ was calculated as described [4]; this relation is equal to that between $\theta_{n} / \theta_{r}$ and $t_{n}$. After rearrangement of the relation between $\theta_{n}$ and $\eta_{n}$ given in [7], it follows that

$$
\frac{\theta_{n}}{\theta_{r}}=\frac{1}{\theta_{r}+\left(1-\theta_{r}\right) \exp -\left[\frac{F \eta_{n}}{R T}\right]}
$$

Calculated relations between $\eta_{n}$ and $\log t_{n}$ are shown in Figs. 1 and 2. $\eta_{n}$ is plotted $u s \log t_{n}$ for $j_{O . T}=$ $10^{-4} \mathrm{~A} / \mathrm{cm}^{2}, D=10^{-5} \mathrm{~cm}^{2} / \mathrm{s}, \theta_{r}=10^{-2}$ and various values of $\theta_{0}$ in Fig. 1. The influence of $j_{0}, T, D$ and $\theta_{r}$ upon the $\eta_{m} / \log t_{n}$ relation are shown in Fig. 2 . In all our calculations $T=298 \mathrm{~K}$, and $p=10^{5} \mathrm{~cm}^{3} / \mathrm{mol}$ were used. The calculation was done by means of a Crank-Nicholson difference scheme with nonlinear boundary conditions[12] on a Burroughs B7600 com-

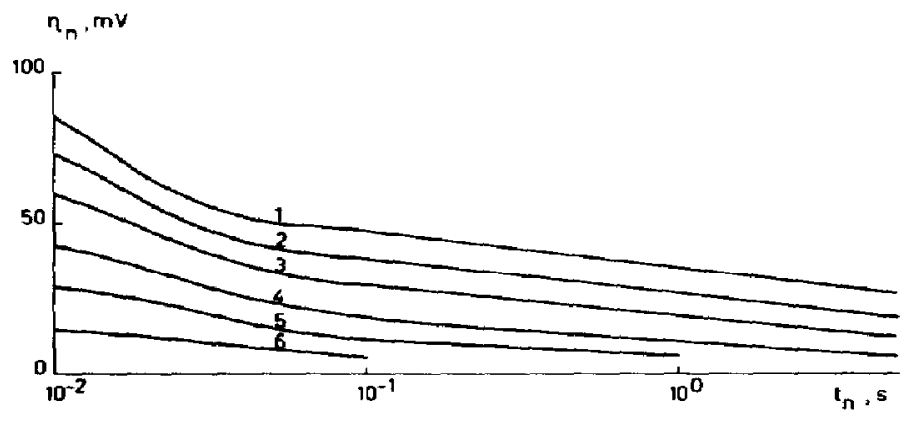

Fig. 1. Potential decay curves for the chlorine formation on open-circuit according to the Volmer-Tafel mechanism at various values of $\theta_{0} / \theta_{r}$ and $\eta_{n}(0)$ and at $j_{0 . T}=10^{-4} \mathrm{~A} / \mathrm{cm}^{2}, \theta_{r}=10^{-2}$ and $D=10^{-5} \mathrm{~cm}^{2} / \mathrm{s}$.

$\begin{array}{ccc}\text { curve } & \theta_{\mathrm{o}} / \theta_{\mathrm{r}} & \eta_{\mathrm{n}}(0) \mathrm{mV} \\ 1 & 80 & 154 \\ 2 & 40 & 108 \\ 3 & 20 & 83 \\ 4 & 8 & 55 \\ 5 & 4 & 36 \\ 6 & 2 & 18\end{array}$




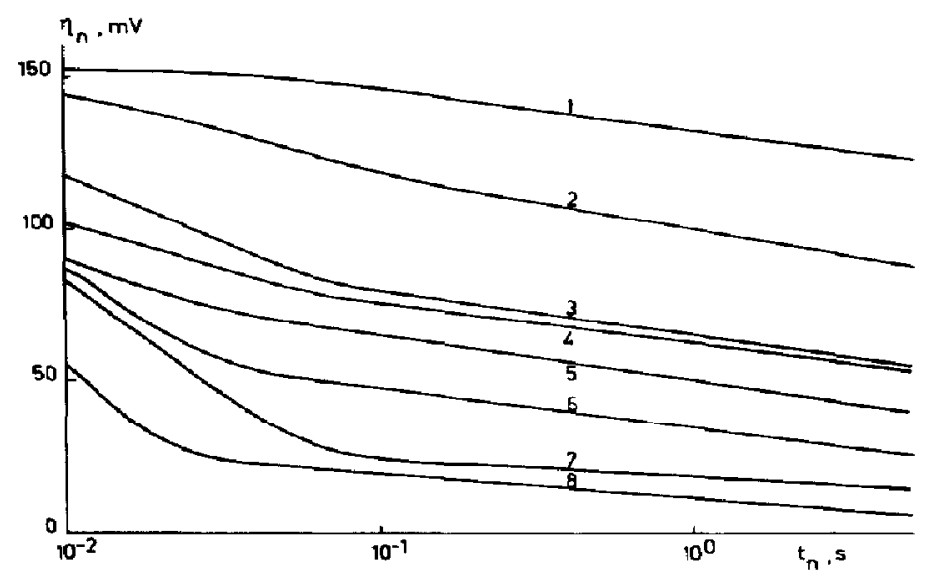

Fig. 2. Potential decay curves for the chlorine formation on open-circuit according to the Volmer-Tafel mechanism at various values of $j_{0, T}, \theta_{,}, \eta_{n}(0)$ and $D$ and for $\theta_{0} / \theta_{r}=80$.

$\begin{array}{ccccc}\text { curve } & j_{0 . \mathrm{T}} \mathrm{A} / \mathrm{cm}^{2} & D \mathrm{~cm}^{2} / \mathrm{s} & \theta_{r^{2}} & \eta_{m}(0) \mathrm{mV} \\ 1 & 10^{-7} & 10^{-5} & 10^{-2} & 154 \\ 2 & 10^{-6} & 10^{-5} & 10^{-2} & 154 \\ 3 & 10^{-3} & 10^{-5} & 10^{-2} & 154 \\ 4 & 10^{-4} & 10^{-5} & 10^{-3} & 115 \\ 5 & 10^{-4} & 10^{-4} & 10^{-2} & 154 \\ 6 & 10^{-4} & 10^{-5} & 10^{-2} & 154 \\ 7 & 10^{-4} & 10^{-6} & 10^{-2} & 154 \\ 8 & 10^{-3} & 10^{-5} & 10^{-2} & 154\end{array}$

puter. The time step size we used was $\Delta t=0.01 \mathrm{~s}$ and for the $x$-coordinate 640 gridpoints from 0 - $\infty$ were taken. In the literature $[2,6,7]$ the rate of the gas evolution or the total volume of gas evolved is given as a function of the time after switching off the polarising current and even so the total volume of gas evolved as a function of the potential on open circuit.

From the calculated relation between $\theta_{n}$ and $t_{n}$ (Fig. 2) and from (1), the relation between $j_{n}, \mathrm{~T}$ and $t_{n}$ was calculated. $\log \left(j_{\mathrm{n}, \mathrm{T}} / j_{0}, \mathrm{~T}\right)$ is plotted $v s \log t_{n}$ in Fig. 3 for various values of $j_{0 . T}$ and for $D=10^{-5} \mathrm{~cm}^{2} / \mathrm{s}$, $\theta_{r}=10^{-2}, \theta_{0}=0.8$ and $\eta_{n}(0)=154 \mathrm{mV}$. Figure 4 shows the relation between $\eta_{n}$ and $j_{0}, \mathrm{~T} q_{\mathrm{T}}$, where $q_{\mathrm{T}}$ is proportional to the total volume of evolved gas during the time $t_{n}$ after switching off the polarising current. This value was determined by graphical integration of the $j_{n, T} / t_{n}$ curve.

The behaviour of the electrode after switching off the polarising current is closely related to the behaviour of the electrode during anodic polarisation, as shows the relation between $\eta_{i}$ and $\eta_{n}(0)$. This relation can be calculated from the theoretical relations between $\eta_{i}$ and $\theta_{i}[9]$ and between $\eta_{n}$ and $\theta_{n}[3]$ with substitution of $\eta_{n}$ by $\eta_{n}(0)$ and of $\theta_{n}$ by $\theta_{0}$.

In Figure $5 \eta_{n}(0)$ is plotted vs $\eta_{i}$ for $\theta_{r}=10^{-2}$, $j_{0, r}=10^{-4} \mathrm{~A} / \mathrm{cm}^{2}$ and for various values of $j_{0, \mathrm{~T}} / j_{0, \mathrm{v}}$. This figure shows that $\eta_{n}(0)=\eta_{i}$ when $j_{0}, \mathbf{r} / j_{0}, \mathbf{v} \rightarrow 0$; this means that in this case the volmer reaction has no influence at all upon the $\eta_{t} / \log j_{i}$ relation which is then completely determined by the Tafel reaction.

To elucidate the rate-determining step of the $\mathrm{Cl}_{2} / \mathrm{Cl}^{-}$reaction, the $\eta_{\mathrm{N}}(0) / \eta_{i}$ relation should be useful. However, the experimental determination of $\eta_{n}(0)$ is often not easily done owing to both the discharge of the jonic double-layer and the sharp decrease of $\eta_{n}$ directly after switching off the polarising current. That means in many cases $\eta_{m}(0)$ cannot be used for elucidation of the reaction mechanism. However, the overpotential on open circuit at more or less arbitrary $t_{n}=1 \mathrm{~s}, \eta_{n}(1)$, appears to be the more useful.

The $\eta_{n}(1) / \eta_{i}$-relation can be deduced from the $\eta_{i} / \theta_{i}$

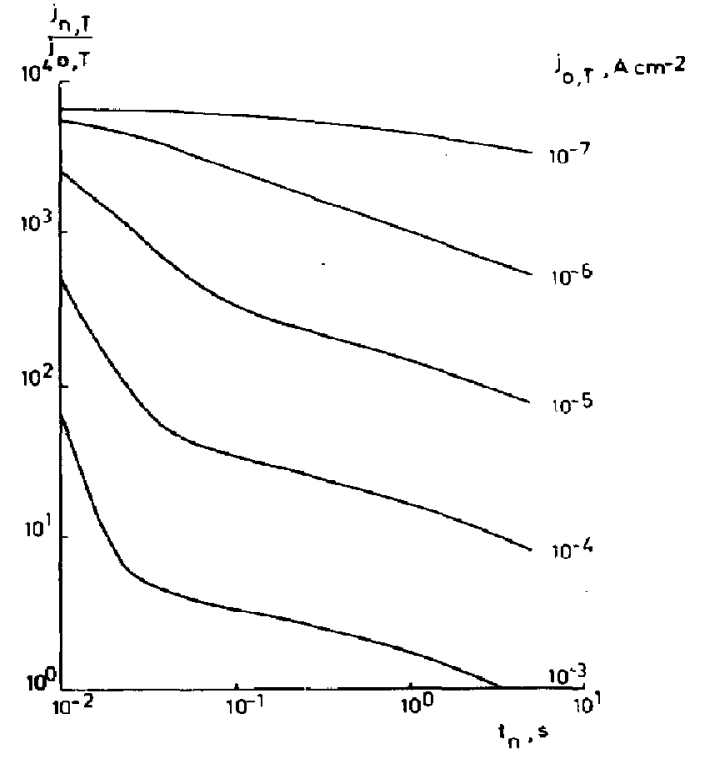

Fig. 3. $j_{n}, \mathrm{~T} / j_{0}$. T $v s$ the time after switching off the polarising current for the chlorine formation according to the Volmer-Tafel mechanism at various values of $j_{0, T}$ and at $D=10^{-5} \mathrm{~cm}^{2} / \mathrm{s}, \theta_{r}=10^{-2}, \theta_{0}=0.8$ and $\eta_{n}(0)=154 \mathrm{mV}$. 


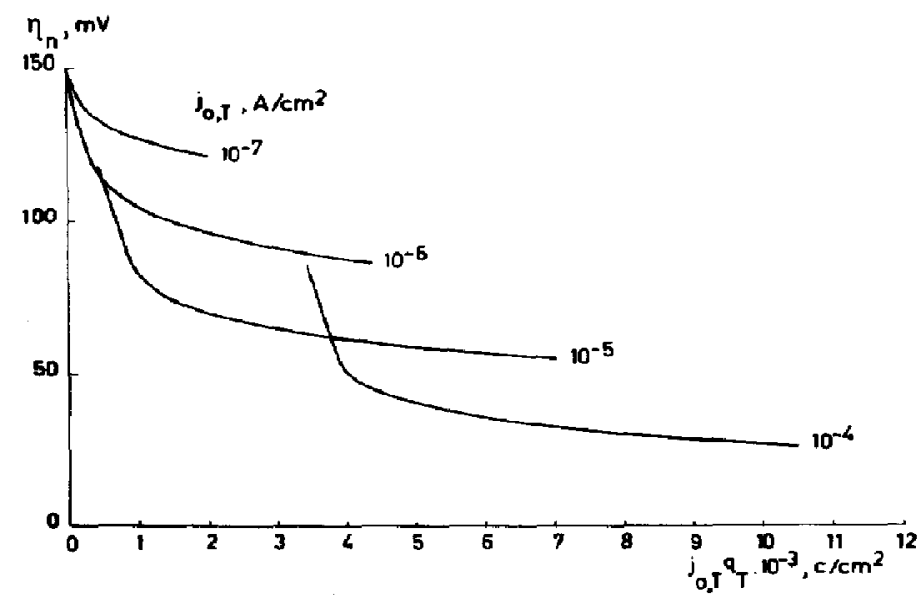

Fig. 4. Relationship between the overvoltage on open-circuit and the charge $j_{0}, T q_{T}$ for the chlorine formation according to the Volmer-Tafel mechanism at various values of $j_{0, T}$ and at $D=10^{-5} \mathrm{~cm}^{2} / \mathrm{s}, \theta_{r}=10^{-2}$, $\theta_{0}=0.8$ and $\eta_{n}(0)=154 \mathrm{mV}$.

$\eta_{n}(0) / \theta_{0}$ and $\eta_{n} / \log t_{n}$ relations with $\theta_{0}=\theta_{i^{-}} \eta_{n}(1) / \eta_{i^{-}}$ relations are also plotted in Fig. 5 for various values of $j_{0 . \tau} / j_{0 . v}$ and for $\theta_{r}=10^{-2}, j_{0, \tau}=10^{-4} \mathrm{~A} / \mathrm{cm}^{2}$ and $D=10^{-5} \mathrm{~cm}^{2} / \mathrm{s}$.

In Figure $6 \quad \eta_{M}(1) / \eta_{i}$ curves are shown for
The potential on open circuit can also be determined by this mechanism. The relation between the degree of coverage and the overpotential on open circuit is rather complex. Assuming that $\alpha_{V}=\alpha_{H}$, it has been deduced [6] that

$$
\theta_{n}=\frac{j_{0, \mathrm{~V}}-j_{0, \mathrm{H}} \exp \left(-\frac{F \eta_{n}}{R T}\right)}{j_{0, \mathrm{~V}}-j_{0, \mathrm{H}} \frac{1-\theta_{r}}{\theta_{r}}+\left(\frac{1-\theta_{r}}{\theta_{r}} j_{0, \mathrm{~V}}-j_{0, \mathrm{H}}\right) \exp \left(-\frac{F \eta_{r}}{R T}\right)} .
$$

$j_{\mathrm{n}, \mathrm{r}} / j_{\mathrm{n}, \mathrm{v}} \rightarrow 0, \theta_{\mathrm{r}}=10^{-2}, c_{\mathrm{r}}=10^{-7} \mathrm{~mol} / \mathrm{cm}^{3}$ and various values of $j_{0, \mathrm{~T}}$ and $D$.

\subsection{Volmer-Heyrovsky mechanism}

This mechanism consists of the Volmer reaction $\left(\mathrm{Cl}^{-} \rightleftharpoons \mathrm{Cl}_{\mathrm{ad}}+e^{-}\right)$and the Heyrovsky reaction $\left(\mathrm{Cl}^{-}+\mathrm{Cl}_{\text {ad }} \rightleftarrows \mathrm{Cl}_{2}+e^{-}\right)$.

Neglecting the discharge of the double-layer as before, the net polarising current on open circuit is zero and the absolute value of the net rate of the Heyrovsky reactions is then equal to that of the Volmer reaction; this means that $j_{\mathbf{H}}=-j_{\mathbf{V}}$.

The rate of the removal of atomic chlorine can be deduced from the rate equation for the anodic and

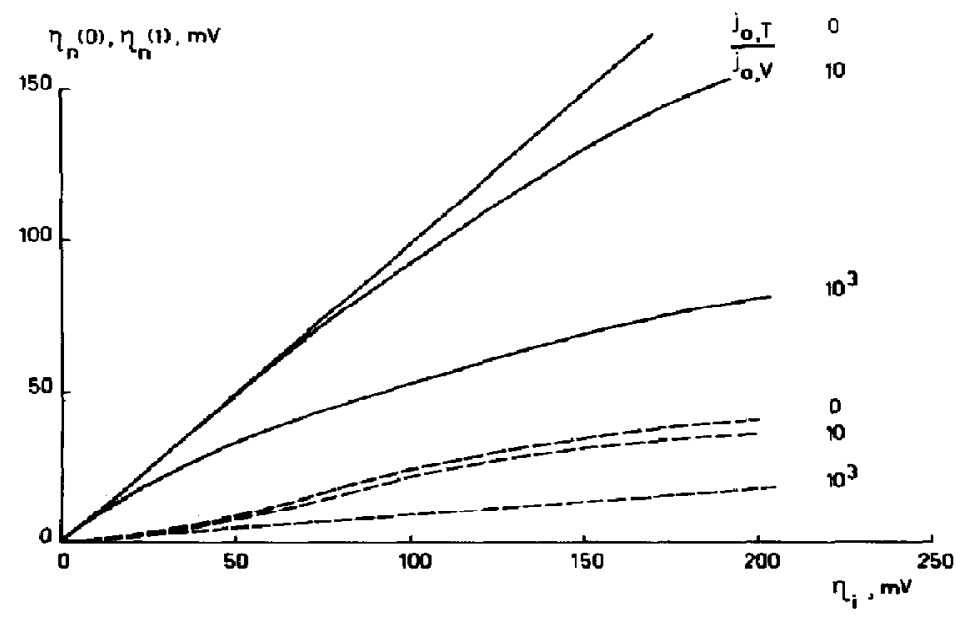

Fig. 5. Relationship between the overvoltage on open-circuit at the moment of switching off the polarising current and the overvoltage on current flow $(-)$ and relationship between the overvoltage on open-circuit at $1 \mathrm{~s}$ after switching of the polarising current and the overvoltage on current-flow (-- - ). Both relationships are given for the chlorine formation according to the Volmer-Tafel mechanism at various values of $j_{\mathrm{o}}, r / j_{0}, \mathrm{~V}$ and at $j_{0, \mathrm{~T}}=10^{-4} \mathrm{~A} / \mathrm{cm}^{2}, \theta_{r}=10^{2}$ and $D=10^{-3} \mathrm{~cm}^{2} / \mathrm{s}$. 


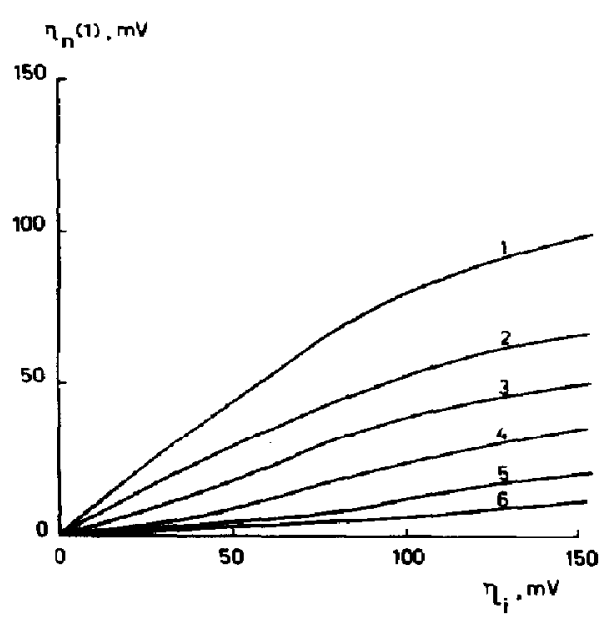

Fig. 6. Overvoltage on open-circuit at $1 \mathrm{~s}$ after switching off the polarising current $v$ s overvoltage on current flow for the chlorine formation according to the Volmer-Tafel mechanism at various values of $j_{0 . T}$ and $D$ and $a t \theta_{p}=10^{-2}$ and $j_{\mathbf{0}, \mathbf{T}} / j_{0, \mathrm{v}} \rightarrow 0$.

$\begin{array}{ccc}\text { curve } & j_{0 .} \mathrm{A} / \mathrm{cm}^{2} & D \mathrm{~cm}^{2} / \mathrm{s} \\ 1 & 10^{-6} & 10^{-5} \\ 2 & 10^{-5} & 10^{-5} \\ 3 & 10^{-4} & 10^{-4} \\ 4 & 10^{-4} & 10^{-5} \\ 5 & 10^{-4} & 10^{-6} \\ 6 & 10^{-3} & 10^{-5}\end{array}$

cathodic Heyrovsky reaction. It follows that

$j_{H}=j_{0, \mathrm{H}}\left(\frac{\theta_{n}}{\theta_{r}} \exp \frac{\alpha_{\mathrm{H}} F \eta_{n}}{R T}-\frac{\left(1-\theta_{n}\right)}{\left(1-\theta_{r}\right)}\right.$

$$
\left.\times \exp -\frac{\left(1-\alpha_{H}\right) F \eta_{n}}{R T}\right)
$$

As in section 2.1 , it is assumed that atomic chlorine diffuses out of the bulk of the electrode material. So, the one-dimensional second equation of Fick can be applied. The boundary conditions used are

$$
\begin{gathered}
c(x, 0)=c_{0} \text { and } x \geqslant 0, \\
c\left(\infty, t_{n}\right)=c_{0} \text { for } t_{n} \geqslant 0, \\
D \frac{\delta c\left(x, t_{n}\right)}{\delta x}=\frac{2 j_{\mathbf{O} . \mathbf{H}}\left(\frac{c\left(0, t_{n}\right)}{c_{r}}\right.}{} \\
\left.\quad \times \exp \frac{F \eta_{n}}{2 R T}-\frac{1-p c\left(0, t_{n}\right)}{1-p c_{r}} \exp -\frac{F \eta_{n}}{2 R T}\right)
\end{gathered}
$$

where $\theta=p c$ and $\alpha_{F}=0.5$. For this case, the relation between $c\left(0, t_{n}\right) / c_{r}$ and $t_{n}$ and that between $\eta_{n}$ and $t_{n}$ were calculated for various $j_{0, H}, j_{0, H} / j_{0}, v, c_{0}, c_{r}$ and $D$ values. The relation between $\theta_{n}$ and $n$ is given by (3) for $j_{0, \mu} / j_{0, \mathrm{v}} \rightarrow 0$ and by (4) for the other $j_{0, \mathrm{v}}$ values.

Calculated $\eta_{n} / \log t_{n}$ curves are shown in Figs. 7 and 8. The results are given in Fig. 7 for $j_{\mathrm{O}, \mathrm{H}}=5 \times 10^{-6}$ $\mathrm{A} / \mathrm{cm}^{2}, j_{0, \mathrm{H}} / j_{0, \mathrm{v}} \rightarrow 0, D=10^{-5} \mathrm{~cm}^{2} / \mathrm{s}, \theta_{\mathrm{r}}=10^{-3}$ and various $\theta_{0}$ values. The influence of $j_{0, H} D$ and $\theta_{r}$ at $j_{0, v} / j_{0, H} \rightarrow 0$ upon the $\eta_{m} / \log t_{n}$ curves are shown in Fig. 8. The effect of $j_{0, w} / j_{0}, v$ is shown in Fig. 9, where $j_{0, H}=5 \times 10^{-6} \mathrm{~A} / \mathrm{cm}^{2}, \quad D=10^{-5} \mathrm{~cm}^{2} / \mathrm{s}$ and $\theta_{r}=10^{-2}$.

Just as for the Volmer-Tafel mechanism, the theoretical relation between the gas evolution rate on open circuit, expressed as the current density $j_{n, H}$ and the time after switching off the polarising current was determined. This relation was obtained from (5) and from the calculated $\eta_{n} / \log t_{n}$ curves. In Fig. $10 \log$

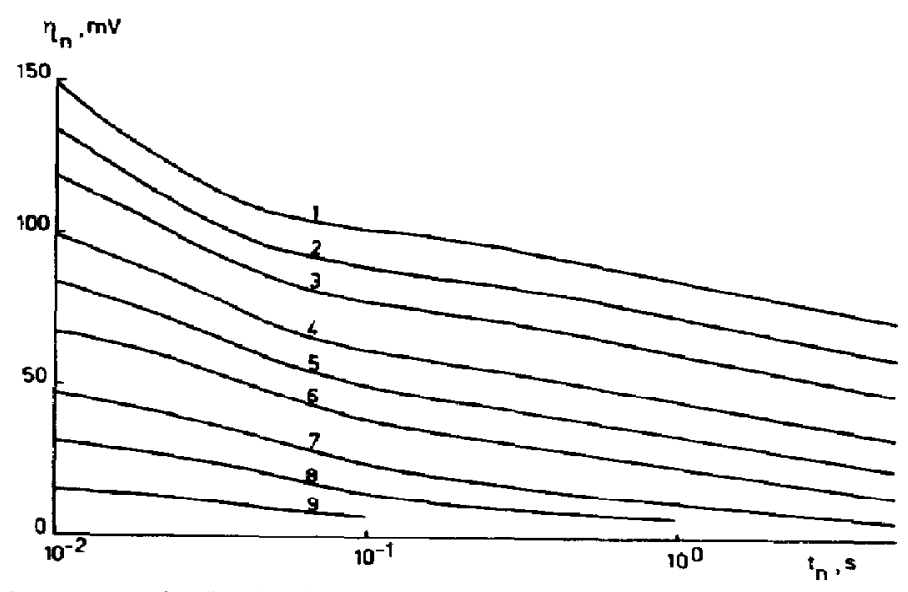

Fig. 7. Potential decay curves for the chlorine formation on open-circuit according to the Volmer-Heyrovsky mechanism at various values of $\theta_{0} / \theta_{r}$ and $\eta_{n}(0) \mathrm{mV}$ and at $j_{0, \mathrm{H}} / j_{0, \mathrm{v}} \rightarrow 0, j_{0, \mathrm{H}}=5 \times 10^{-6} \mathrm{~A} / \mathrm{cm}^{2}, \theta_{\mathrm{r}}=10^{-3}$ and $D=10^{-5} \mathrm{~cm}^{2} / \mathrm{s}$.

$\begin{array}{ccc}\text { curve } & \theta_{\delta} / \theta_{r} & \eta_{n}(0) \mathrm{mV} \\ 1 & 800 & 213 \\ 2 & 400 & 167 \\ 3 & 200 & 142 \\ 4 & 80 & 115 \\ 5 & 40 & 96 \\ 6 & 20 & 78 \\ 7 & 8 & 54 \\ 8 & 4 & 36 \\ 9 & 2 & 18\end{array}$




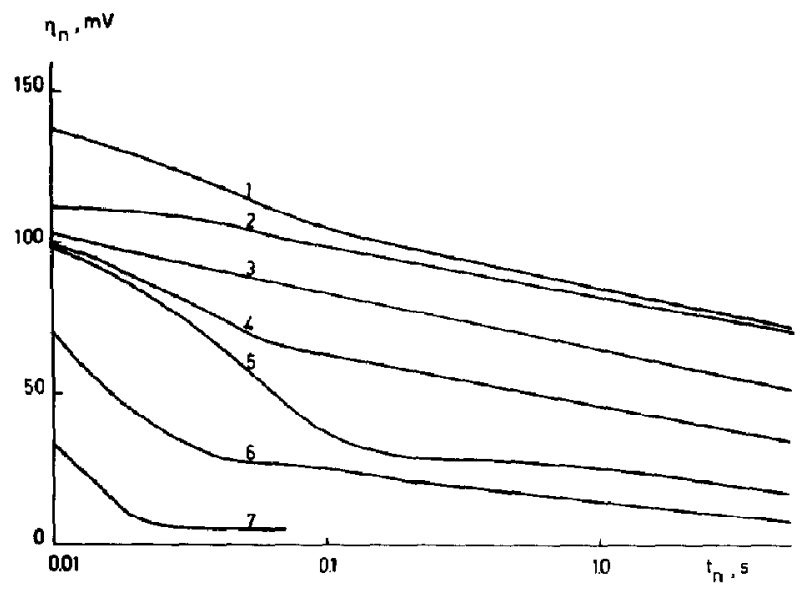

Fig. 8. Potential decay curves for the chlorine formation on open-circuit according to the Volmer-Heyrovsky mechanism at various values of $j_{0, H}, \theta_{n}, \eta_{n}(0)$ and $D$ and at $j_{0, \mathrm{H}} / j_{0, \mathrm{v}} \rightarrow 0$ and $\theta_{\mathrm{a}} / \theta_{r}=80$.

$\begin{array}{ccccc}\text { curve } & j_{0, \mathrm{H}} \mathrm{A} / \mathrm{cm}^{2} & D \mathrm{~cm}^{2} / \mathrm{s} & \theta_{r} & \eta_{n}(0) \mathrm{mV} \\ 1 & 5 \times 10^{-6} & 10^{-5} & 10^{-2} & 154 \\ 2 & 5 \times 10^{-7} & 10^{-5} & 10^{-3} & 115 \\ 3 & 5 \times 10^{-6} & 10^{-4} & 10^{-3} & 115 \\ 4 & 5 \times 10^{-6} & 10^{-5} & 10^{-3} & 115 \\ 5 & 5 \times 10^{-6} & 10^{-6} & 10^{-3} & 115 \\ 6 & 5 \times 10^{-5} & 10^{-5} & 10^{-3} & 115 \\ 7 & 5 \times 10^{-4} & 10^{-5} & 10^{-3} & 115\end{array}$

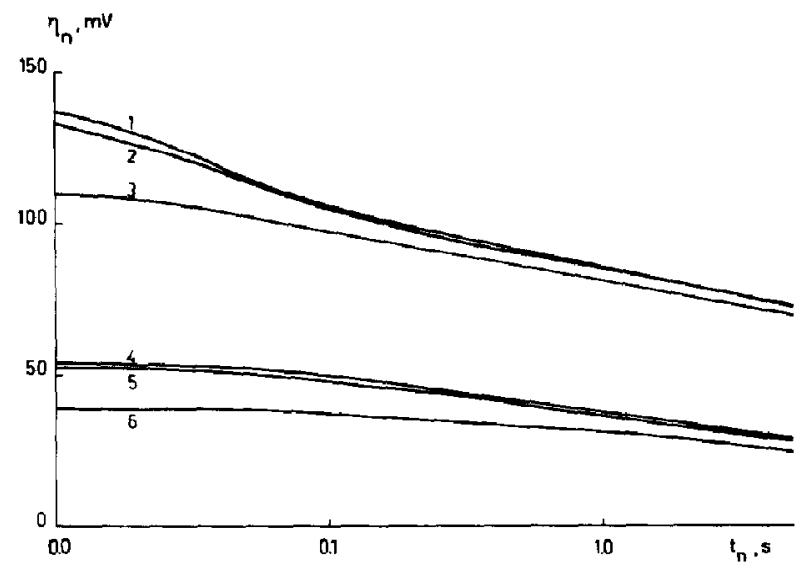

Fig. 9. Potential decay curves for the chlorine formation on open-circuit according to the Volmer-Heyrovsky mechanism at various values of $j_{0, H} / j_{c}, v_{n} \theta_{0} / \theta_{r}$ and $\eta_{n}(0)$ and for $\theta_{r}=10^{-2}$ and $D=10^{-5} \mathrm{~cm}^{2} / \mathrm{s}$ and $j_{0 . \mathbf{H}}=5 \times 10^{-6} \mathrm{~A} / \mathrm{cm}^{2}$.

$\begin{array}{cccc}\text { curve } & j_{0 . \mu} / j_{\mathrm{O} . \mathrm{V}} & \theta_{0} / \theta_{r} & \eta_{n}(0) \mathrm{mV} \\ 1 & 0 & 80 & 154 \\ 2 & 10^{-3} & 80 & 145 \\ 3 & 10^{-2} & 80 & 113 \\ 4 & 0 & 8 & 55 \\ 5 & 10^{-2} & 8 & 53 \\ 6 & 10^{-1} & 8 & 40\end{array}$




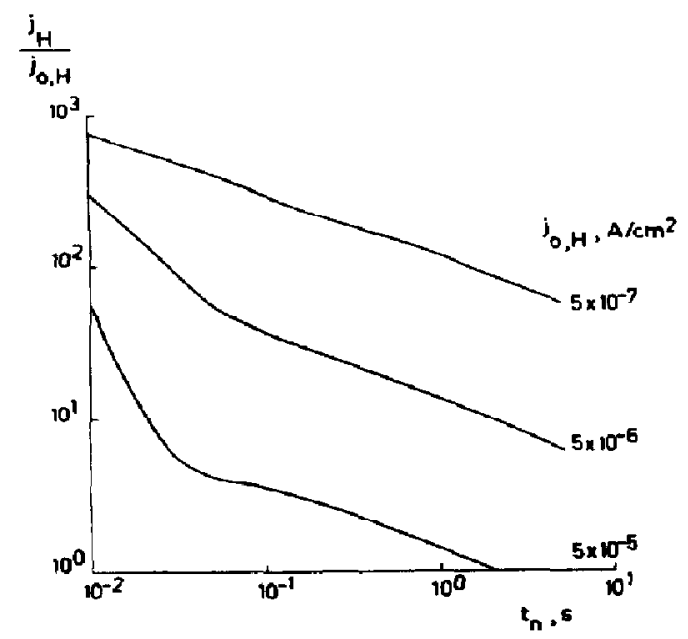

Fig. $10 . j_{m . \mathrm{H}} / j_{0 . \mathrm{H}}$ os the time after switching off the polarising current for the chlorine formation according to the Volmer-Heyrovsky mechanism at various values of $j_{O, H}$ and at $D=10^{-5} \mathrm{~cm}^{2} / \mathrm{s}, \theta_{r}=10^{-3}, \theta_{0}=0.08$ and $J_{0, H} / j_{0, V} \rightarrow 0$.

$\left(j_{n, \mathbf{H}} / j_{0, \mathrm{H}}\right)$ is plotted $v s \log t_{n}$ for $j_{0, \mathrm{H}} / j_{0, \mathrm{v}} \rightarrow 0$, $D=10^{-5} \mathrm{~cm}^{2} / \mathrm{s}, \theta_{r}=10^{-3}, \theta_{0}=0.08$ and various values of $j_{0 . \mathrm{H}}$. The $\eta_{n} / j_{0 .} q_{H}$ relation was obtained on the same way as that for the Tafel reaction. This relation is shown in Fig. 11 for the parameters of Fig. 10.

Analogous to the Tafel treatment, relations between $\eta_{l}$ and $\eta_{n}(0)$ and between $\eta_{i}$ and $\eta_{m}(1)$ can be derived. In Fig. 12, both $\eta_{n}(0)$ and $\eta_{n}(1)$ are plotted $v s \eta_{i}$ for $j_{0, H} / j_{0}, w$. In Fig, $13 \eta_{n}(1) / \eta_{i}$ curves are given for $\theta_{r}=10^{-}, j_{0 . \mathrm{H}} / j_{0} . \mathrm{v} \rightarrow 0$ and various values of $j_{0 . \mathrm{H}}$ and D.

\section{DISCUSSION}

3.1 Usefuiness of the investigation of decay behaviour

By comparing the experimental with the theoretical $\eta_{n} / \log t_{n}$ curves, in principle it is possible to elucidate the reaction mechanism of the chlorine evolution. This becomes much easier when the experimental $\eta_{i} / \log j_{i}$ curves are thoroughly known.

For the two discussed mechanisms the $\eta_{n} / \log t_{n}$ as well as the $\log \left(j_{n} / j_{0}\right) / \log t_{n}$ curves at $\eta_{n}>20 \mathrm{mV}$ and $\eta_{n}$ (0) $-\eta_{n}>20 \mathrm{mV}$ are practically linear for a wide range of exchange current densities, diffusion coefficients, degrees of coverage at the reversible potential and of overpotentials during anodic polarisation. From sections 2.1 and 2.2 it follows that the absolute values of the slopes of the $\eta_{n} / \log t_{n}$ and of $\log \left(j_{n} / j_{0}\right) /$ $\log t_{n}$ curve at $25^{\circ} \mathrm{C}$ are about $14 \mathrm{mV}$ and 0.5 , respectively, for the Tafel reaction as the $\mathrm{T}$ ate determining step and about $20 \mathrm{mV}$ and 0.5 , respectively, for the Heyrovsky reaction as the rate-determining step. These results agree with those in the literature[4].

The slopes of the $\log \left(j_{n, T} / j_{0}, \mathrm{~T}\right) / \log t_{n}$ and of the $\log$ $\left(j_{n, \mathbf{H}} / j_{0, \mathbf{H}}\right) / \log t_{n}$ curves are equal. Therefore, the determination of the rate of the chlorine evolution on open circuit as a function of the time after switching off the polarising current reveals nothing about the mechanism. In the literature $\eta_{n}(0)$ has been determined by linear extrapolation of the relation between $\eta_{n}$ and the quantity of hydrogen evolved on open circuit [7] and between $\eta_{n}^{-1}$ and $t_{n}^{1 / 2}[6]$. From the results in 2.1 and 2.2 it can be deduced that both methods of determining $\eta_{n}(0)$ can give erroneous results. Only at a slow potential decay during the first period after switching off the polarising current experimental determination is possible. In this case, the use of the $\eta_{n} \log t_{n}$ relation is preferred. Moreover, $\eta_{n}(0)$ is equal to $\eta_{i}$ for both mechanisms if the Volmer reaction has no effect upon the $\eta_{i} / \log j_{i}$ relation.

\subsection{Application to earlier experimental results}

Triacia, Solomons and Bockris $[10]$ investigated the chlorine evolution on graphite in molten lithium chloride. From the slope of the $\eta_{i} / \log j_{i}$ curve and the stoichiometric number they concluded that chlorine is formed according to the Volmer-Tafel mechanism, where the Tafel reaction is the rate-determining step. The experimental anodic decay curves were explained by assuming a decrease of the adsorption psendo-

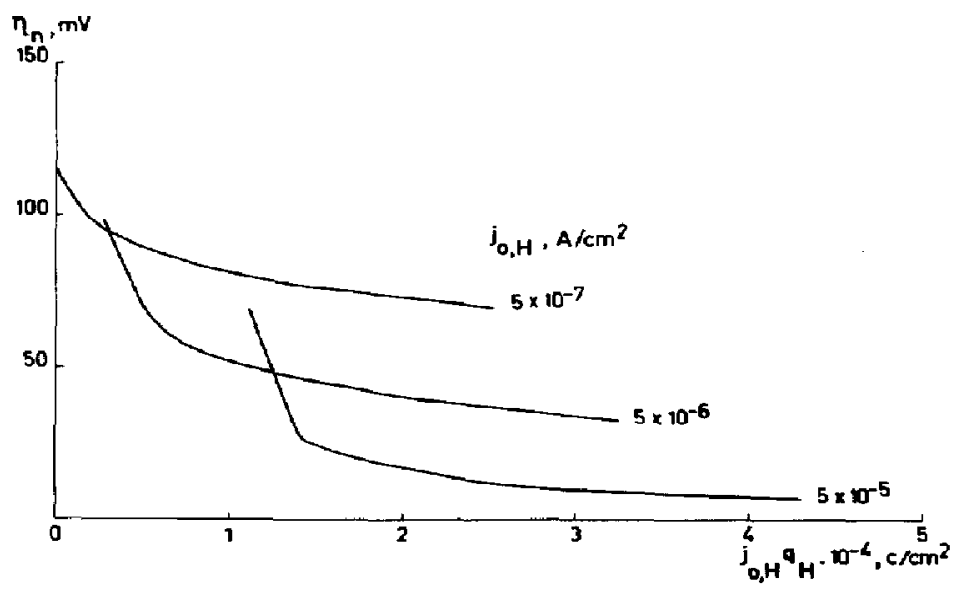

Fig. 11. Relationship between the overpotential on open-circuit and the charge $j_{0,} q_{H}$ for the chlorine formation according to the Volmer-Heyrovsky mechanism at various values of $j_{0, H}$ and at $j_{0 . n} / j_{0 .} \rightarrow 0$, $D=10^{-5} \mathrm{~cm}^{2} / \mathrm{s}, \theta_{\mathrm{r}}=10^{-3}$ and $\theta_{0}=0.08$. 


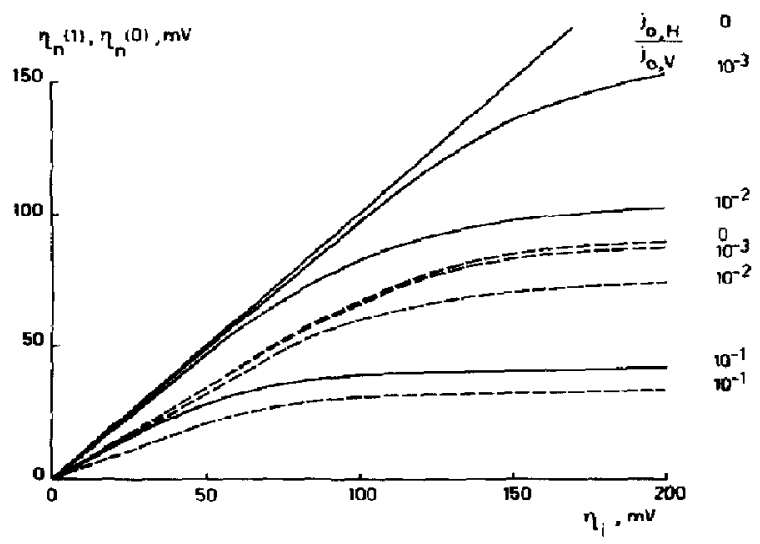

Fig. 12. Relationship between the overvoltage on open-circuit at the moment of switching off the polarising current and the overvoltage on current flow (- - ) and relationship between the overvoltage on open-circuit at $1 \mathrm{~s}$ after switching off the polarising current and the overvoltage on current flow (-- - -). Both relationships are given for the chlorine formation according to the Volmer-Heyrovsky mechanism at $j_{0, \mathrm{H}}=5 \times 10^{-6} \mathrm{~A} / \mathrm{cm}^{2}, D=10^{-5} \mathrm{~cm}^{2} / \mathrm{s}, \theta_{v}=10^{-7}$ and at various values of $j_{0}, \mathbf{H} / j_{0}, \mathbf{v}$.

capacitance with increasing potential and a change variation of heat of adsorption with the degree of coverage by atomic chlorine. From a comparison of the experimental with the theoretical $\eta_{n} / \log t_{n}$ relation, Tilak and Conway concluded that the experimental curves can be explained by assuming a non-activated adsorption of chlorine atoms with strong repulsive

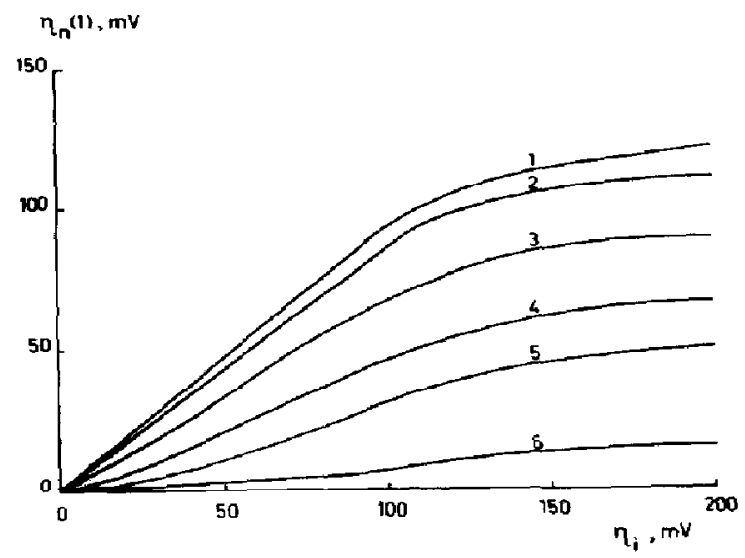

Fig. 13. Overvoltage on open-circuit at $1 \mathrm{~s}$ after switching off the polarising current $v$ s averpotential on current flow for the chlorine formation according to the Volmer-Heyrovsky mechanism at various values of $j_{0, H}$ and $D$ and at $\theta_{0}=0.08$, $0_{r}=10^{-2}$ and $j_{0, H} / j_{0, v} \rightarrow 0$.

$\begin{array}{ccc}\text { curve } & j \mathrm{HA}_{\mathrm{H}} \mathrm{\textrm {cm } ^ { 2 }} & D \mathrm{~cm}^{2} / \mathrm{s} \\ 1 & 5 \times 10^{-7} & 10^{-5} \\ 2 & 5 \times 10^{-0} & 10^{-4} \\ 3 & 5 \times 10^{-6} & 10^{-5} \\ 4 & 5 \times 10^{-6} & 10^{-6} \\ 5 & 5 \times 10^{-5} & 10^{-5} \\ 6 & 5 \times 10^{-4} & 10^{-5}\end{array}$

interaction between adsorbed chlorine atoms (3).

The experimental anodic $\eta_{n} / \log t_{\mathrm{H}}$ curves at $t_{n}>10^{-4} \mathrm{~s}$ have a slope of $-2.3(R T / 4 F)[10]$. The theoretical slope $h_{\mathrm{T}}$ of the $\eta_{n} / \log t_{n}$ curves is $-14 \mathrm{mV}$ for the Tafel reaction as rate-determining reaction. At $\eta_{n}>20 \mathrm{mV}, \eta_{n}(0)-\eta_{n}>20 \mathrm{mV}$ and at $25^{\circ} \mathrm{C}$, this value of $h_{\mathrm{T}}$ is equal to $-2.3(R T / 4 F)$.

Consequently, corrected for temperature, both slopes are equal. Moreover, the shape of the experimental $\eta_{n} / \log t_{n}$ curve [10] agrees with that of the curve in Fig. 2 for $j_{n, T}=10^{-7} \mathrm{~A} / \mathrm{cm}^{2}$. Only the time scale of the two curves is different. This difference can be eliminated by using a higher exchange-current density and diffusion coefficient in calculating the theoretical $n_{n} / \log t_{n}$ curve.

The formation of chlorine during anodic polarisation and on open circuit at graphite electrodes in aqueous sodium chloride hydrochloride solution was studied by Janssen and Hoogland $[6,9,11]$. They found that chlorine is formed according to the Volmer-Heyrovsky mechanism. For an aged graphite electrode the Heyrovsky reaction is the ratedetermining step, whereas for a new graphite electrode both the Volmer and the Heyrovsky reactions determine the relation between potential and current density.

From the theory (2.1 and 2.2) it follows that especially the relation between $\eta_{m}$ and $t_{n}$ is important for elucidation of the mechanism. Unfortunately, this relation was not determined during the first period onto about $3 \mathrm{~s}$ after switching off the anodic current [6]. From Fig. 4, reference[6] it can be deduced that for an aged electrode in the investigated range of $t_{n}, \eta_{n}$ is smaller than $20 \mathrm{mV}$ and the slope $h$ of the $\eta_{n} / \log t_{n}$ curve is $-7.5 \mathrm{mV}$ and for a new electrode the slope $h$ is - $56 \mathrm{mV}$ at $\eta_{n}>25 \mathrm{mV}$. Both electrodes were previously polarised with an anodic current density of $45 \mathrm{~mA} / \mathrm{cm}^{2}$ at $25^{\circ} \mathrm{C}$. These slopes can be explained by both the Volmer-Tafel and the Volmer-Heyrovsky mechanisms, although the first mechanism is preferred. This preference is based mainly on the slope of $-56 \mathrm{mV}$, which agrees with the theoretical one for the Volmer-Tafel mechanism where it is mainly the atomic chlorine present on the outer surface of the electrode that determines the $\eta_{n} / \log t_{n}$ curve[4]. Addition experiments, especially at $t_{n}<37 \mathrm{~s}$, are necessary if better founded conclusions are to be obtained.

The potential decay behaviour was also used to elucidate the mechanism of the chlorine formation on ruthenium oxide-titanium oxide electrodes and on ruthenium electrodes[4]. In reference[4] $\eta_{n}(0)-\eta_{n}$ have been plotted $v s \log t_{n}$; the questionable value of this relation lies in the great uncertainty of the value of $\eta_{n}(0)$. However, this has no influence upon the slope of the $\eta_{n} / \log t_{n}$ curve. Since the back reaction affects the $\eta_{n} / \log t_{n}$ curve only at very low $\eta_{n}$, viz $\eta_{n}<20 \mathrm{mV}$, the conclusions drawn in reference[4] are correct.

\section{REFERENCES}

1. P. Rüetschi, Shlarchuk and Amlie, J. electrochem. Soc. 107, 325 (1960); 108, 377 (1961).

2. B. E. Conway and P. L. Bourgault, Trans. Faraday Soc. 5B, 593 (1962).

3. B. V. Tilak and B. E. Conway, Electrachim. Acta 21, 745 (1976). 
4. L. J. J. Janssen, L. M. C. Starmans, J. G. Visser and $E$. Barendrecht, Electrochim. Acta 22, 1093 (1977).

5. C. A. Knorr, $Z$. Electrochem. 57, 599 (1953).

6. L. J. J. Janssen and J. G. Hoogland, Electrochim. Acta 15, 1667 (1970).

7. R. Clamroth and C. A. Knorr, Z. Electrochem. 57, 399 (1953).

8. K. J. Bachmant, Ber. der Bursenyesellschaft 71, 1073 (1967).
9. L. J. J. Janssen and J. G. Hoogland, Electrochim. Acta 15, $941(1970)$.

10. J. Triacia, B. Solomons and J. O'M. Bockris, Electrochim. Acta 13, 1949 (1968)

11. L. J. J. Janssen, Thesis Eindhoven 1968.

12. D. U. von Rosenberg, Method for the Numerical Solution of Particle Differential Equations, p. 75. Elsevier, New York (1969). 